Pacific Journal of Mathematic 


\title{
LINEAR TRANSFORMATIONS WHICH PRESERVE HERMITIAN AND POSITIVE SEMIDEFINITE OPERATORS
}

\author{
JoHn de PILLIS
}

\begin{abstract}
Let $\mathfrak{U}$ and $\mathfrak{B}$ represent the full algebras of linear operators on the finite-dimensional unitary spaces $\mathscr{H}$ and $\mathscr{K}$, respectively. The symbol $\mathscr{L}(\mathfrak{X}, \mathfrak{B})$ will denote the complex space of all linear maps from $\mathfrak{A}$ to $\mathfrak{B}$. This paper concerns itself with the study of the following two cones in $\mathscr{L}(\mathfrak{U}, \mathfrak{B})$ :

(i) the cone $\mathscr{C}$ of all $T \in \mathscr{L}(\mathfrak{A}, \mathfrak{B})$ which send hermitian operators in $\mathfrak{A}$ to hermitian operators in $\mathfrak{B}$, and

(ii) the subcone $\mathscr{C}^{+}($of $\mathscr{C})$ of all $T \in \mathscr{L}(\mathfrak{A}, \mathfrak{B})$ which send positive semidefinite operators in $\mathfrak{A}$ to positive semidefinite operators in $\mathfrak{B}$.
\end{abstract}

In our main results, we characterize the transformations in the cone $\mathscr{C}$ (Theorem 2.1) and present a structure theorem concerning the transformations in the cone $\mathscr{C}^{+}$(Theorem 2.3). Identifying operators in the algebras $\mathfrak{U}$ and $\mathfrak{B}$ with appropriate square matrices, we may summarize Theorem 2.1 by saying that any and every linear transformation $\boldsymbol{T}$ which preserves hermitian matrices is of the form $\boldsymbol{T}: A \rightarrow \sum \alpha_{i} X_{i}^{*} A^{t} X_{i}$, where each $\alpha_{i}$ is a real scaler, and each $X_{i}$ is a certain rectangular matrix depending on $\boldsymbol{T} ; X_{i}^{*}$ and $A^{t}$ represent the conjugate transpose and the transpose of matrices $X_{i}$ and $A$, respectively. Theorem 2.3 says that the cone of positive semidefinitepreserving transformations $\mathscr{C}^{+}$"generates" or spans all of $\mathscr{L}(\mathfrak{H}, \mathfrak{B})$ in the sense that any $T$ in $\mathscr{L}(\mathfrak{A}, \mathfrak{B})$ can be written

$$
\boldsymbol{T}=\left(\boldsymbol{K}_{1}-\boldsymbol{K}_{2}\right)+i\left(\boldsymbol{K}_{3}-\boldsymbol{K}_{4}\right),
$$

where $i^{2}=-1$, and each $\boldsymbol{K}_{i}$ is an element of $\mathscr{C}^{+}$.

1. Preliminaries. $L(\mathscr{K}, \mathscr{H})$ denotes the space of linear transformations from the Hilbert space $\mathscr{K}$ to the Hilbert space $\mathscr{H}$. We define:

1 (a). $(x \times y)$-the dyad transformation, an element of $L(\mathscr{K}, \mathscr{H})$, is defined for fixed $x \in \mathscr{H}$ and $y \in \mathscr{K}$ by: $(x \times y)(z)=(z, y) x$ for all $z \in \mathscr{K}$, where $(z, y)$ is the inner product of $z$ with $y$. As it turns out, $(x, y)=\operatorname{tr}((x \times y))$, the trace of $(x \times y)$. If $A \in \mathfrak{A}(=(L(\mathscr{H}, \mathscr{H}))$ and $B \in \mathfrak{B}(=L(\mathscr{K}, \mathscr{K}))$, then $(A(x) \times B(y))=A(x \times y) B^{*}$.

1 (b). $P_{x}$-denotes the orthogonal projection onto the subspace spanned by $x$, i.e., for $(x, x)=1$, we have $P_{x}=(x \times x)$. 
1 (c). $[A, B]$-is the inner product defined on $\mathfrak{A}$ (resp. $\mathfrak{B})$ by setting $[A, B]=\operatorname{tr}\left(B^{*} A\right)$ for all $A, B \in \mathfrak{A}$ (resp. $\left.\mathfrak{B}\right)$ where $B^{*}$ is the Hilbert space adjoint of $B$, and $\operatorname{tr}(\cdot)$ is the trace functional on $\mathfrak{A}$ (resp. $\mathfrak{B})$. More generally, $L(\mathscr{K}, \mathscr{H})$ becomes a Hilbert space once we define the inner product $[A, B]=\operatorname{tr}\left(B^{*} A\right)$ for all $A, B \in L(\mathscr{K}, \mathscr{H})$. Consequently, for $w_{1}, w_{2} \in \mathscr{H}$, and $u_{1}, u_{2} \in \mathscr{K}$, so that $\left(w_{1} \times u_{1}\right)$ and $\left(w_{2} \times u_{2}\right)$ belong to $L(\mathscr{K}, \mathscr{H})$, we have

$$
\begin{aligned}
{\left[\left(w_{1} \times u_{1}\right),\left(w_{2} \times u_{2}\right)\right] } & =\operatorname{tr}\left(\left(w_{2} \times u_{2}\right)^{*}\left(w_{1} \times u_{1}\right)\right) \\
& =\operatorname{tr}\left(\left(u_{2} \times w_{2}\right)\left(w_{1} \times u_{1}\right)\right) \\
& =\operatorname{tr}\left(\left(w_{1}, w_{2}\right)\left(u_{2} \times u_{1}\right)\right) \\
& =\left(w_{1}, w_{2}\right)\left(u_{2}, u_{1}\right) .
\end{aligned}
$$

1 (d). $(A][B)$ - the dyad transformation, an element of $\mathscr{L}(\mathfrak{B}, \mathfrak{A})$, is defined for fixed transformations $A \in \mathfrak{A}$ and $B \in \mathfrak{B}$ by $(A][B) \cdot C=$ $[C, B] A$, for all $C$ in $B$. As in $1(\mathrm{a}) .,[A, B]=\operatorname{tr}((A][B))$, the trace of $(A][B)$.

1 (e). $\quad \mathfrak{A} \otimes \mathfrak{B}$-the tensor product of algebras $\mathfrak{A}$ and $\mathfrak{B}$, consists of sums of elements of the form $A \otimes B$, where $A \in \mathfrak{A}$ and $B \in \mathfrak{B}$ [2, Chapter 16]. The symbol $(A \otimes B)^{0}$ will denote the element $B \otimes A$, and can be linearly extended to any element of $\mathfrak{A} \otimes \mathfrak{B}$.

1 (f). $\left[A_{1} \otimes B_{1}, A_{2} \otimes B_{2}\right]$-the inner product which gives the algebra $\mathfrak{A} \otimes \mathfrak{B}$ a Hilbert space structure, is defined by

$$
\left[A_{1} \otimes B_{1}, A_{2} \otimes B_{2}\right]=\left[A_{1}, A_{2}\right] \cdot\left[B_{1}, B_{2}\right]
$$

for all $A_{1}, A_{2} \in \mathfrak{A}$, and all $B_{1}, B_{2} \in \mathfrak{B}$.

$1(\mathrm{~g}) . \quad \mathscr{I}(\boldsymbol{T})$ - the element of $\mathfrak{A} \otimes \mathfrak{B}$ which is defined for each $\boldsymbol{T}$ in $\mathscr{L}(\mathfrak{A}, \mathfrak{B})$ by $\left[\mathscr{J}(\boldsymbol{T}), A^{*} \otimes B\right]=[\boldsymbol{T}(A), B]$, for all $A \in \mathfrak{A}, B \in \mathfrak{B}$. This equation also defines $\mathscr{F}$ as a linear transformation, sending the space $\mathscr{P}(\mathfrak{A}, \mathfrak{B})$ to the algebra $\mathfrak{A} \otimes \mathfrak{B}$.

1 (h). $\overline{\mathscr{C}}$-the space of all linear functionals on $\mathscr{H}$. For each $x \in \mathscr{H}$, we define the functional $\bar{x} \in \overline{\mathscr{H}}$ by $\bar{x}(y)=(y, x)$ for all $y \in \mathscr{H}$. Moreover, these are the only elements of $\overline{\mathscr{H}}$. An inner product is defined on $\overline{\mathscr{H}}$ by setting $(\bar{x}, \bar{y})=(y, x)$ for all $\bar{x}, \bar{y} \in \overline{\mathscr{H}}$. Thus, $(\bar{x}, \bar{y})=\overline{(x, y)}$, the complex conjugate of $(y, x)$.

1 (i). $A^{t}$-the transpose of the operator $A$, is the linear operator on $\overline{\mathscr{H}}$ defined by $A^{t}(\bar{y})(x)=\bar{y}(A(x))$, for all $\bar{y} \in \overline{\mathscr{H}}$, and all $x \in \mathscr{H}$ 
[1, p. 103]. From this it follows that $(x \times y)^{t}=(\bar{y} \times \bar{x})$. If $\bar{A}$ is defined to be $\left(A^{*}\right)^{t}$, then $(\overline{x \times y})=(\bar{x} \times \bar{y})$ and $\bar{A}(\bar{x})=\overline{A(x)}$. From this we see that for all $A \in \mathfrak{A}, \bar{A}^{*}=A^{t}$. In fact, set $A=(x \times y)$ for $x, y \in \mathfrak{A}$. Then

$$
\bar{A}^{*}=(\overline{x \times y})^{*}=(\bar{x} \times \bar{y})^{*}=(\bar{y} \times \bar{x})=(\overline{y \times x})=(x \times y)^{t}=A^{t} .
$$

Hence, by linear extension, $\bar{A}^{*}=A^{t}$ for all $A \in \mathfrak{A}$.

1 (j). $\quad L(\overline{\mathscr{K}}, \mathscr{H})$-is spanned by the dyads $(x \times \bar{y})$, where $x \in \mathscr{H}$ and $\bar{y} \in \overline{\mathscr{K}}$. In this context, we identify the transformation $A \otimes B$ with the transformation $C \rightarrow A C B^{t}$ for all $C \in L(\overline{\mathscr{K}}, \mathscr{H})$, where $A \in \mathfrak{A}(=L(\mathscr{H}, \mathscr{H}))$ and $B \in \mathfrak{B}(=L(\mathscr{K}, \mathscr{K})$. Behind this identification is the isomorphism $\phi: \mathscr{H} \otimes . \overline{\mathscr{K}} \rightarrow L(\mathscr{K}, \mathscr{H})$ defined by $\phi(x \otimes y)=$ $(x \times \bar{y})$ for all $x \in \mathscr{H}, y \in \mathscr{K}$. If for each $A \in \mathfrak{A}, B \in \mathfrak{B}$ we define the linear transformation $\boldsymbol{O}_{A, B}: L(\overline{\mathscr{K}}, \mathscr{\mathscr { C }}) \rightarrow L(\overline{\mathscr{K}}, \mathscr{X})$ by $\boldsymbol{O}_{A, B}(C)=$ $A C B^{t}$ for all $C \in L(\overline{\mathscr{K}}, \mathscr{H})$, then $A \otimes B$ corresponds to $O_{A, B}$ in the sense that $\phi \circ(A \otimes B) \circ \phi^{-1}=\boldsymbol{O}_{A, B}$. In fact, we have

$$
\begin{aligned}
\left(\dot{\phi} \circ(A \otimes B) \circ \dot{\phi}^{-1}(x \times \bar{y})\right. & =\phi(A \otimes B(x \otimes y)) & & \text { definition of } \dot{\phi}^{-1} \\
& =\phi(A(x) \otimes B(y)) & & \text { definition of } A \otimes B \\
& =(A(x) \times \overline{B(y)}) & & \text { definition of } \phi \\
& =(A(x) \times \bar{B}(\bar{y})) & & \text { from } 1(\mathrm{i}) . \\
& =A(x \times \bar{y}) \bar{B}^{*} & & \text { from } 1(\mathrm{a}) . \\
& =A(x \times \bar{y}) B^{t} & & \text { since } \bar{B}^{*}=B^{t}, \text { see } 1(\mathrm{i}) . \\
& =\boldsymbol{O}_{A, B}((x \times \bar{y})) & & \text { definition of } \boldsymbol{O}_{A, B} .
\end{aligned}
$$

For convenience, however, we shall treat $A \otimes B$ as though it were actually equal to the concrete linear transformation $\boldsymbol{O}_{A, B}=A(\cdot) B^{t}$. In so doing, we have

$$
(x \times y)][(u \times v)=(x \times u) \otimes(\bar{y} \times \bar{v})
$$

for vectors $x, y, u, v$ in (not necessarily the same) Hilbert space.

The linear transformation $\mathscr{J}$ (see $1(\mathrm{~g})$.) will prove to be of fundamental importance. For this reason, we isolate some of its properties in

Proposition 1.1. (1) $\mathscr{I}(B][A)=A^{*} \otimes B$ for all $A \in \mathfrak{A}, B \in \mathfrak{B}$.

(2) $\mathscr{J}(\boldsymbol{T})=\sum_{i} E_{i}^{*} \otimes \boldsymbol{T}\left(E_{i}\right)$ for any and every orthonormal basis $\left\{E_{i}\right\}$ for $\mathfrak{A}$.

(3) If $\boldsymbol{T}\left(A^{*}\right)=\boldsymbol{T}(A)^{*}$ for all $A \in \mathfrak{A}$ (i.e., if $T \in \mathscr{C}$ ), then $\mathscr{J}(\boldsymbol{T})=$ $\sum_{i} \boldsymbol{T}^{*}\left(F_{i}\right) \otimes F_{i}^{*}$ for any orthonormal basis $\left\{F_{i}\right\}$ for $\mathfrak{B}$.

(4) If $\boldsymbol{T}\left(A^{*}\right)=\boldsymbol{T}(A)^{*}$ for all $A \in \mathfrak{A}$, then $\mathscr{J}\left(\boldsymbol{T}^{*}\right)=\mathscr{J}(\boldsymbol{T})^{0}$. 
(5) $\mathscr{I}$ is an isometric isomorphism from the Hilbert space $\mathscr{L}(\mathfrak{A}, \mathfrak{B})$ onto the Hilbert algebra $\mathfrak{A} \otimes \mathfrak{B}$.

Proof. From the definition $1(g)$. of $\mathscr{I}$, we have

$$
\begin{array}{rlrl}
{[\mathscr{J}(B][A), C \otimes D]} & =\left[(B][A)\left(C^{*}\right), D\right] & & \\
& =\left[C^{*}, A\right][B, D] & & \text { from } 1(\mathrm{~d}) . \\
& =\left[A^{*}, C\right][B, D] & \\
& =\left[A^{*} \otimes B, C \otimes D\right] & & \text { from } 1(\mathrm{f}) .
\end{array}
$$

for all $A, C \in \mathfrak{A}$ and all $B, D \in \mathfrak{B}$. This implies Part (1).

Now let $\left\{E_{i}\right\}$ be any orthonormal (o.n.) basis for $\mathfrak{A}$. If $\boldsymbol{T}=(B][A)$ for $A \in \mathfrak{U}$ and $B \in \mathfrak{B}$, then

$$
\begin{array}{rlr}
\sum_{i} E_{i}^{*} \otimes \boldsymbol{T}\left(E_{i}\right) & =\sum_{i} E_{i}^{*} \otimes(B][A)\left(E_{i}\right) & \\
& =\sum_{i}\left[E_{i}, A\right] E_{i}^{*} \otimes B & \\
& =\sum_{i}\left[A^{*}, E_{i}^{*}\right] E_{i}^{*} \otimes B & \\
& =A^{*} \otimes B & \\
& =\mathscr{J}(B][A) . & \text { from 1 (d). }
\end{array}
$$

The dyads $(B][A), A \in \mathfrak{A}, B \in \mathfrak{B}$, span the space $\mathscr{L}(\mathfrak{A}, \mathfrak{B})$, so that (using linearity of $\mathscr{J}$ ) for all $\boldsymbol{T} \in \mathscr{L}(\mathfrak{A}, \mathfrak{B}), \mathscr{J}(\boldsymbol{T})=\sum_{i} E_{i}{ }^{*} \otimes \boldsymbol{T}\left(E_{i}\right)$, which establishes Part (2).

Part (3) follows from (2) and (4) inasmuch as if $\mathscr{I}\left(\boldsymbol{T}^{*}\right)=\mathscr{I}(\boldsymbol{T})^{0}$, then $\sum \boldsymbol{T}^{*}\left(F_{i}\right) \otimes F_{i}^{*}=\left(\sum F_{i}^{*} \otimes \boldsymbol{T}^{*}\left(F_{i}\right)\right)^{0}=\mathscr{I}\left(\boldsymbol{T}^{*}\right)^{0}=\mathscr{I}(\boldsymbol{T})$

But Part (4) obtains, since for all $A \in \mathfrak{A}, B \in \mathfrak{B}$,

$$
\begin{array}{rlrl}
{\left[\mathscr{J}\left(\boldsymbol{T}^{*}\right), A \otimes B\right]} & =\left[\boldsymbol{T}^{*}\left(A^{*}\right), B\right] & & \text { definition } 1(\mathrm{~g}) . \text { of } \mathscr{I} \\
& =\left[\boldsymbol{T}(B)^{*}, A\right] & & \\
& =\left[\boldsymbol{T}\left(B^{*}\right), A\right] & & \text { if and only if } \boldsymbol{T}\left(B^{*}\right)=\boldsymbol{T}(B)^{*} \\
& =[\mathscr{F}(\boldsymbol{T}), B \otimes A] & & \text { definition } 1(\mathrm{~g}) . \text { of } \mathscr{J} \\
& =\left[\mathscr{I}(\boldsymbol{T})^{0}, A \otimes B\right] . &
\end{array}
$$

That is, $\mathscr{I}\left(\boldsymbol{T}^{*}\right)=\mathscr{I}(\boldsymbol{T})^{0}$ and Part (4) is proven.

As for demonstrating Part (5), observe that for all $A_{1}, A_{2} \in \mathfrak{A}$, and $B_{1}, B_{2} \in \mathfrak{B}$,

$$
\begin{aligned}
{\left[\mathscr{J}\left(B_{1}\right]\left[A_{1}\right), \mathscr{I}\left(B_{2}\right]\left[A_{2}\right)\right] } & =\left[A_{1}^{*} \otimes B_{1}, A_{2}^{*} \otimes B_{2}\right] \quad \text { from Part }(1) \\
& =\left[A_{1}^{*}, A_{2}^{*}\right] \operatorname{tr}\left(\left(B_{1}\right]\left[B_{2}\right)\right) \quad \text { from } 1(\mathrm{~d}) . \text { and } 1(\mathrm{f}) . \\
& =\operatorname{tr}\left(\left(B_{1}\right]\left[A_{1}\right) \cdot\left(B_{2}\right]\left[A_{2}\right)^{*}\right) \\
& =\left[\left(B_{1}\right]\left[A_{1}\right),\left(B_{2}\right]\left[A_{2}\right)\right] .
\end{aligned}
$$


By linear extension on each argument of the inner product, we have that for all $\boldsymbol{T}_{1}, \boldsymbol{T}_{2} \in \mathscr{L}(\mathfrak{A}, \mathfrak{B})$,

$$
\left[\mathscr{J}\left(\boldsymbol{T}_{1}\right), \mathscr{J}\left(\boldsymbol{T}_{2}\right)\right]=\left[\boldsymbol{T}_{1}, \boldsymbol{T}_{2}\right]
$$

so that $\mathscr{I}$ is an isometry from $\mathscr{L}(\mathfrak{U}, \mathfrak{B})$ to $\mathfrak{A} \otimes \mathfrak{B}$. From Part (1) it is easy to see that $\mathscr{J}$ is also an onto transformation as well, since the algebra $\mathfrak{A} \otimes \mathfrak{B}$ is spanned by elements of the form $A^{*} \otimes B$. This completes the proof of Proposition 1.1.

Our next result establishes a necessary and sufficient condition for a transformation in $\mathscr{L}(\mathfrak{U}, \mathfrak{B})$ to be in the cone $\mathscr{C}$.

Proposition 1.2. A transformation $T \in \mathscr{L}(\mathfrak{A}, \mathfrak{B})$ is in $\mathscr{C}$ if and only if $\mathscr{J}(\boldsymbol{T})$ is hermitian.

Proof. Recall that $\mathscr{I}$ maps $\mathscr{L}(\mathfrak{A}, \mathfrak{B})$ (isometrically) onto $\mathfrak{A} \otimes \mathfrak{B}$, which has been identified as the algebra of linear operators on the Hilbert space $L(\overline{\mathscr{K}}, \mathscr{H})$ (see $1(\mathrm{j}))$. Now for all $A \in \mathfrak{A}, B \in \mathfrak{B}$,

(a) $\left[\mathscr{I}(\boldsymbol{T})^{*}, A \otimes B\right]=\overline{\left.\mathscr{I}(\boldsymbol{T}), A^{*} \otimes B^{*}\right]}$

$$
=\overline{\left.T(A), B^{*}\right]}
$$

definition $1(\mathrm{~g})$ of $\mathscr{I}$

$$
=\left[\boldsymbol{T}(A)^{*}, B\right]
$$

where (a) and (c) follow from the properties of the inner product, viz., $\overline{[Y, Z]}=\left[Y^{*}, Z^{*}\right]$ for all operators $Y$ and $Z$. Now,

$$
\left[\boldsymbol{T}(A)^{*}, B\right]=\left[\boldsymbol{T}\left(A^{*}\right), B\right] \quad \text { for all } A \in \mathfrak{A}, B \in \mathfrak{B},
$$

if and only if $\boldsymbol{T}(A)^{*}=\boldsymbol{T}\left(A^{*}\right)$ for all $A \in \mathfrak{A}$. Finally, $\left[\boldsymbol{T}\left(A^{*}\right), B\right]$ is equal to $[\mathscr{J}(T), A \otimes B]$, so that for all $A \in \mathfrak{A}, B \in \mathfrak{B}$,

$$
\left[\mathscr{J}(\boldsymbol{T})-\mathscr{J}(\boldsymbol{T})^{*}, A \otimes B\right]=0
$$

if and only if $\boldsymbol{T}\left(A^{*}\right)=\boldsymbol{T}(A)^{*}$. This completes the proof.

REMARK. We have just shown that $\boldsymbol{T} \in \mathscr{L}(\mathfrak{A}, \mathfrak{B})$ preserves hermitian operators $(\boldsymbol{T} \in \mathscr{C})$ if and only if $\mathscr{J}(\boldsymbol{T})$ is hermitian. It is not unreasonable to suspect that $\boldsymbol{T}$ preserves positive semidefinite (psd) operators $\left(\boldsymbol{T} \in \mathscr{C}^{+}\right)$if and only if $\mathscr{I}(\boldsymbol{T})$ is psd. However, this conjecture is false, for if $\mathfrak{A}=L(\mathscr{H}, \mathscr{H})$, and if $\mathfrak{B}=L(\mathscr{K}, \mathscr{K})$, then for any multiplicative transformation $\boldsymbol{T} \in \mathscr{L}(\mathfrak{H}, \mathfrak{B}) \quad(\boldsymbol{T}(A B)=$ $\boldsymbol{T}(A) \boldsymbol{T}(B)$ ), we have $\boldsymbol{T} \in \mathscr{C}^{+}$; but $\mathscr{J}(\boldsymbol{T})$ will always have some negative eigenvalues. For a specific example choose $\mathfrak{A}=\mathfrak{B}=L(\mathscr{H}, \mathscr{H})$, the algebra of operators on $\mathscr{H}$. Let $\boldsymbol{T} \in \mathscr{L}(\mathfrak{A}, \mathfrak{B})$ be the identity transformation $\boldsymbol{T}(A)=A$ for all $A \in \mathfrak{A}$. Surely $\boldsymbol{T} \in \mathscr{C}^{+}$. Now choose the o.n. basis $\left\{e_{1}, e_{2}, \cdots, e_{n}\right\}$ for $\mathscr{C}$; then $\left\{\left(e_{i} \times e_{j}\right): i, j=1,2, \cdots, n\right\}$ is an o.n. basis for $\mathfrak{A}$ so that from Proposition 1.1 Part (2), we have 


$$
\mathscr{J}(\boldsymbol{T})=\sum\left(e_{i} \times e_{j}\right)^{*} \otimes\left(e_{i} \times e_{j}\right)=\sum\left(e_{j} \times e_{i}\right) \otimes\left(e_{i} \times e_{j}\right) .
$$

The situation may be represented by the following diagram:

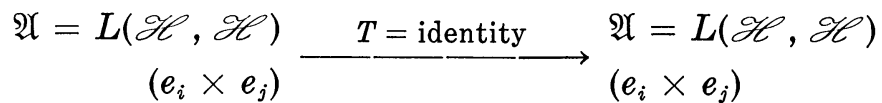

$$
\begin{aligned}
& \begin{aligned}
L(\overline{\mathscr{C}}, \mathscr{H}) \\
\left(e_{p} \times \bar{e}_{q}\right)
\end{aligned} \stackrel{\mathscr{I}(T)=\underline{\text { transpose }} \longrightarrow}{\longrightarrow} \begin{array}{l}
L(\overline{\mathscr{C}}, \mathscr{H}) \\
\left(e_{q} \times \bar{e}_{p}\right) .
\end{array}
\end{aligned}
$$

From 1(i) and 1(j) we conclude that $\mathscr{F}(\boldsymbol{T})\left(\left(e_{p} \times \bar{e}_{q}\right)\right)=\left(e_{q} \times \bar{e}_{p}\right)$ for $\left(e_{p} \times \bar{e}_{q}\right), p, q=1,2, \cdots, n$, in the space $L(\mathscr{\mathscr { H }}, \mathscr{H})$. That is, if $\boldsymbol{T}$ is the identity operator on the Hilbert algebra $L(\mathscr{H}, \mathscr{H})$, then $\mathscr{I}(\boldsymbol{T})$ is the transpose operator on the Hilbert space $L(\overline{\mathscr{C}}, \mathscr{H})$. It is easy to see that vectors of the form $\left(e_{p} \times \bar{e}_{q}\right)-\left(e_{q} \times \bar{e}_{p}\right)$ in $L(\mathscr{\mathscr { C }}, \mathscr{\mathscr { C }})$ are eigenvectors for $\mathscr{J}(\boldsymbol{T})$ corresponding to the eigenvalue $-1 . \mathscr{I}(\boldsymbol{T})$ (which is hermitian due to Proposition 1.2), is therefore not a psd operator on the Hilbert space $L(\overline{\mathscr{C}}, \mathscr{H})$.

2. The main results. We present a structure theorem which characterizes elements of the cone $\mathscr{C}$.

THEOREM 2.1. Suppose that $\boldsymbol{T} \in \mathscr{C} \subset \mathscr{L}(\mathfrak{A}, \mathfrak{B}) . \quad \mathscr{J}(\boldsymbol{T})$ is selfadjoint by Proposition 1.2, with spectral resolution $\sum_{i} \alpha_{i} \mathscr{P}\left(X_{i}\right)$, where $\alpha_{i}$ is real, $\mathscr{P}\left(X_{i}\right)=\left(X_{i}\right]\left[X_{i}\right)$ is the orthogonal one-dimensional projection on the unit vector $X_{i} \in L(\overline{\mathscr{K}}, \mathscr{H})$, and the $X_{i}^{\prime}$ s form an o.n. basis for $L(\mathscr{\mathscr { K }}, \mathscr{H})$. Let $A \in \mathfrak{U}:$ then

$$
\boldsymbol{T}(A)^{t}=\sum_{i} \alpha_{i} X_{i}^{*} A X_{i} .
$$

Proof. For any $x \in \mathscr{H}$ and $y \in \mathscr{K}$,

$$
\begin{aligned}
& {\left[\boldsymbol{T}\left(P_{x}\right), P_{y}\right]=\left[\mathscr{J}(\boldsymbol{T}), P_{x} \otimes P_{y}\right]} \\
& =\sum_{i}\left[\alpha_{i}\left(X_{i}\right]\left[X_{i}\right),(x \times x) \otimes(y \times y)\right] \quad \text { from } 1(\mathrm{~b}) \\
& =\sum_{i}\left[\alpha_{i}\left(X_{i}\right]\left[X_{i}\right),(x \times \bar{y})\right][(x \times \bar{y})] \quad \text { from } 1(j) \\
& =\sum_{i} \alpha_{i} \operatorname{tr}\left((x \times \bar{y}][x \times \bar{y}) \cdot\left(X_{i}\right]\left[X_{i}\right)\right) \quad \text { from } 1(\mathrm{c}) \\
& =\sum_{i} \alpha_{i}\left[X_{i},(x \times \bar{y})\right]\left[(x \times \bar{y}), X_{i}\right] \\
& =\sum_{i} \alpha_{i} \operatorname{tr}\left((\bar{y} \times x) X_{i}\right) \operatorname{tr}\left(X_{i}^{*}(x \times \bar{y})\right) \\
& =\sum_{i} \alpha_{i} \operatorname{tr}\left(\left(\bar{e} \times X_{i}^{*}(x)\right) \operatorname{tr}\left(X_{i}^{*}(x \times \bar{y})\right) \quad\right. \text { since } \\
& (\bar{y} \times x) X_{i}=\bar{y} \times X_{i}^{*}(x) ; \quad \text { see } 1(\mathrm{a})
\end{aligned}
$$




$$
=\sum_{i} \alpha_{i}\left(\bar{y}, X_{i}^{*}(x)\right)\left(X_{i}^{*}(x), \bar{y}\right)
$$

Now for $w_{1}, w_{2} \in \mathscr{C}$ and $u_{1}, u_{2} \in \mathscr{K}$, we have that

$$
\left(u_{2}, u_{1}\right)\left(w_{1}, w_{2}\right)=\left[\left(w_{1} \times u_{1}\right),\left(w_{2} \times u_{2}\right)\right]
$$

so (8) becomes

$$
\begin{aligned}
& =\sum_{i} \alpha_{i}\left[\left(X_{i}^{*}(x) \times X_{i}^{*}(x)\right),(\bar{y} \times \bar{y})\right] \\
& =\sum_{i}\left[\alpha_{i} X_{i}^{*}(x \times x) X_{i},\left(P_{y}\right)^{t}\right] .
\end{aligned}
$$

Since the transpose is a self-adjoint operator, equation (10) becomes

$$
=\sum_{i}\left[\alpha_{i}\left(X_{i}^{*} P_{x} X_{i}\right)^{t}, P_{y}\right]
$$

Thus, for every $x \in \mathscr{H}$ and every $y \in \mathscr{K}$,

$$
\left[\boldsymbol{T}\left(P_{x}\right)-\left(\sum_{i} \alpha_{i} X_{i}^{*} P_{x} X_{i}\right)^{t}, P_{y}\right]=0 .
$$

But then,

$$
\mathbb{T}\left(P_{x}\right)=\left(\sum \alpha_{i} X_{i}^{*} P_{x} X_{i}\right)^{t}
$$

for all $P_{x} \in \mathfrak{2}$. Since the transpose operator squared is the identity, we may apply it to both sides of the last equation to obtain

$$
\boldsymbol{T}\left(P_{x}\right)^{t}=\sum \alpha_{i} X_{i}^{*} P_{x} X_{i}
$$

for all $P_{x} \in \mathfrak{A}$. This result extends from the set of one dimensional orthogonal projections $P_{x}$ to hermitian operators; this, in turn, extends to arbitrary operators of $\mathfrak{A}$. Thus, the theorem is proved.

REMARK. Suppose the dimension of $\mathscr{Z}=n$ and the dimension of $\mathscr{K}=m$, where $\mathscr{H}$ and $\mathscr{K}$ are the underlying Hilbert spaces for the operator algebras $\mathfrak{U}$ and $\mathfrak{B}$, respectively. Relative to certain ordered bases for $\mathscr{H}$ and $\mathscr{K}$, each operator of $\mathfrak{A}$ and $\mathfrak{B}$ is identified with a certain square matrix. The o.n. basis vectors $X_{i}$ of $L(\overline{\mathscr{K}}, \mathscr{H})$ are then realized as certain $n \times m$ matrices; the operator $X_{i}^{*}$ is identified with the $m \times n$ conjugate transpose matrix of $X_{i}$. Thus, Theorem 2.1 may be interpreted as saying that any linear transformation $\boldsymbol{T}$, sending the full matrix algebra $\mathfrak{A}$ to the full matrix algebra $\mathfrak{B}$ is of the form

$$
\boldsymbol{T}(A)=\left(\sum_{i} \alpha_{i} X_{i}^{*} A X_{i}\right)^{t}
$$

for certain real scalars $\alpha_{i}$ and certain $n \times m$ matrices $X_{i}$, if and only 
if $\boldsymbol{T}$ preserves hermitian matrices. Equivalently,

$$
\begin{aligned}
\boldsymbol{T}(A) & =\left(\sum_{i} \alpha_{i} X_{i}^{*} A X_{i}\right)^{t} \\
& =\sum_{i} \alpha_{i} X_{i}^{t} A^{t}\left(X_{i}^{*}\right)^{t} \\
& =\sum_{i} \alpha_{i} Y_{i}^{*} A^{t} Y_{i}
\end{aligned}
$$$$
\text { setting } Y_{i}=\left(X_{i}^{*}\right)^{t}
$$

for certain real scalars $\alpha_{i}$ and certain $n \times m$ matrices $Y_{i}$ depending on $\boldsymbol{T}$, characterizes those transformations $\boldsymbol{T}: \mathfrak{A} \rightarrow \mathfrak{B}$ which preserve hermitian matrices.

Corollary 2.2. Let $\boldsymbol{T} \in \mathscr{L}(\mathfrak{A}, \mathfrak{B})$ where $\mathscr{J}(\boldsymbol{T})$ is psd in $\mathfrak{A} \otimes \mathfrak{B}$. Then $\boldsymbol{T} \in \mathscr{C}^{+} \subset \mathscr{L}(\mathfrak{A}, \mathfrak{B})$.

Proof. Since $\mathscr{I}(\boldsymbol{T})$ is psd in $\mathfrak{A} \otimes \mathfrak{B}, \mathscr{I}(\boldsymbol{T})$ has spectral resolution $\sum \alpha_{i} \mathscr{P}\left(X_{i}\right)$ where the scalars $\alpha_{i}$ are nonnegative, $\mathscr{P}\left(X_{i}\right)$ is the orthogonal one-dimensional projection onto $X_{i} \in L(\mathscr{\mathscr { K }}, \mathscr{H})$ and the $X_{i}$ 's form an o.n. basis for $L(\overline{\mathscr{K}}, \mathscr{H})$. Since $\mathscr{I}(\boldsymbol{T})$ is psd, it is, a fortiori, self-adjoint, so that $\boldsymbol{T}$ is at least an element of the cone $\mathscr{C}$ (Proposition 1.2). But this gives us sufficient leverage to employ the representation of Theorem 2.1. Hence, $\boldsymbol{T}(\cdot)^{t}=\sum \alpha_{i} X_{i}^{*}(\cdot) X_{i}$ where the $\alpha_{i}$ 's are nonnegative scalars. In order to show that $\boldsymbol{T}$ sends psd operators to psd operators (i.e., $\boldsymbol{T} \in \mathscr{C}^{+}$), it is (necessary and) sufficient to show that $\boldsymbol{T}$ sends one-dimensional orthogonal projections $P_{x}$ to psd operators; to do this, it is (necessary and) sufficient to show that the operator $\boldsymbol{T}(\cdot)^{t}$ sends these projections $P_{x}$ to psd operators. But

$$
\boldsymbol{T}\left(P_{x}\right)^{t}=\sum \alpha_{i}\left(X_{i}^{*} P_{x} X_{i}\right)
$$

from Theorem 2.1. Observe that each term $X_{i}^{*} P_{x} X_{i}=\left(P_{x} X_{i}\right)^{*}\left(P_{x} X_{i}\right)$ is psd, and hence, so is $\sum_{i} \alpha_{i} X_{i}^{*} P_{x} X_{i}$, the sum of nonnegative multiples of these psd terms. The proof is done.

We come to our final theorem which tells us that the cone $\mathscr{C}^{+}$ "generates" the space $\mathscr{L}(\mathfrak{A}, \mathfrak{B})$ in much the same way that the cone of psd operators (in $\mathfrak{A}$, say) "generates" $\mathfrak{A}$.

Theorem 2.3. Suppose $\boldsymbol{T} \in \mathscr{L}(\mathfrak{A}, \mathfrak{B})$. Then for some $\boldsymbol{K}_{1}, \boldsymbol{K}_{2}, \boldsymbol{K}_{3}$, $\boldsymbol{K}_{4} \in \mathscr{C}^{+}$,

$$
\boldsymbol{T}=\left(\boldsymbol{K}_{1}-\boldsymbol{K}_{2}\right)+i\left(\boldsymbol{K}_{3}-\boldsymbol{K}_{4}\right)
$$

where $i^{2}=-1$

Proof. $\mathscr{I}(\boldsymbol{T})$, an element of the algebra $\mathfrak{A} \otimes \mathfrak{B}$ can be decomposed as follows: 
$(*)$

$$
\mathscr{J}(\boldsymbol{T})=\left(\boldsymbol{U}_{1}-\boldsymbol{U}_{2}\right)+i\left(\boldsymbol{U}_{3}-\boldsymbol{U}_{4}\right),
$$

where each of the $\boldsymbol{U}_{i}$ 's is psd in $\mathfrak{A} \otimes \mathfrak{B}$. Proposition 1.1, Part (5), tells us that $\mathscr{I}: \mathscr{L}(\mathfrak{A}, \mathfrak{B}) \rightarrow \mathfrak{A} \otimes \mathfrak{B}$ is an isometry. Since the (vector space) dimensions of $\mathscr{L}(\mathfrak{A}, \mathfrak{B})$ and $\mathfrak{A} \otimes \mathfrak{B}$ agree, $\mathscr{J}$ is, in fact, oneto-one and onto; thus, $\mathscr{I}^{-1}$ exists as a well-defined linear operator. Applying $\mathscr{I}^{-1}$ to $(*)$ yields

$$
\boldsymbol{T}=\left[\mathscr{J}^{-1}\left(\boldsymbol{U}_{1}\right)-\mathscr{I}^{-1}\left(\boldsymbol{U}_{2}\right)\right]+i\left[\mathscr{J}^{-1}\left(\boldsymbol{U}_{3}\right)-\mathscr{I}^{-1}\left(\boldsymbol{U}_{4}\right)\right] .
$$

Now let $\boldsymbol{K}_{i}=\mathscr{I}^{-1}\left(\boldsymbol{U}_{i}\right), i=1,2,3,4$. Corollary 2.2 forces us to conclude that $\boldsymbol{K}_{i} \in \mathscr{C}^{+}$since $\mathscr{I}\left(\boldsymbol{K}_{i}\right)=\boldsymbol{U}_{i}$ is psd. Thus, for any $\boldsymbol{T} \in \mathscr{L}(\mathfrak{A}, \mathfrak{B})$

$$
\boldsymbol{T}=\left(\boldsymbol{K}_{1}-\boldsymbol{K}_{2}\right)+i\left(\boldsymbol{K}_{3}-\boldsymbol{K}_{4}\right)
$$

where each $\boldsymbol{K}_{i} \in \mathscr{C}^{+} \subset \mathscr{L}(\mathfrak{A}, \mathfrak{B})$.

\section{BIBLIOGRAPHY}

1. Hoffman and Kunze, Lineara algebra, Prentice Hall, New York, 1961.

2. Mostow, Sampson and Meyer, Fundamental structures of algebra, McGraw Hill, New York, 1963.

Received April 4, 1966. This work was supported in part by an NSF grant. Portions of this paper derived from the author's doctoral thesis (1965) which was written under Professor J. Feldman at the University of California, Berkeley.

UNIVERSITY OF CALIFORNIA

RIVERSIDE, CALIFORNIA 



\section{PACIFIC JOURNAL OF MATHEMATICS}

\section{EDITORS}

\section{H. ROYDEN}

Stanford University

Stanford, California

J. P. JANS

University of Washington

Seattle, Washington 98105

\section{J. DUGUNDJI}

Department of Mathematics

Rice University

Houston, Texas 77001

RICHARD ARENS

University of California

Los Angeles, California 90024

ASSOCIATE EDITORS

E. F. BECKENBACH

B. H. NeumanN

F. WoLF

K. YosidA

\section{SUPPORTING INSTITUTIONS}

\author{
UNIVERSITY OF BRITISH COLUMBIA \\ CALIFORNIA INSTITUTE OF TECHNOLOGY \\ UNIVERSITY OF CALIFORNIA \\ MONTANA STATE UNIVERSITY \\ UNIVERSITY OF NEVADA \\ NEW MEXICO STATE UNIVERSITY \\ OREGON STATE UNIVERSITY \\ UNIVERSITY OF OREGON \\ OSAKA UNIVERSITY \\ UNIVERSITY OF SOUTHERN CALIFORNIA
}

\author{
STANFORD UNIVERSITY \\ UNIVERSITY OF TOKYO \\ UNIVERSITY OF UTAH \\ WASHINGTON STATE UNIVERSITY \\ UNIVERSITY OF WASHINGTON \\ AMERICAN MATHEMATICAL SOCIETY \\ CHEVRON RESEARCH CORPORATION \\ TRW SYSTEMS \\ NAVAL ORDNANCE TEST STATION
}




\section{Pacific Journal of Mathematics}

\section{Vol. 23, No. 1 \\ March, 1967}

M. J. C. Baker, A spherical Helly-type theorem ................... 1

Robert Morgan Brooks, On locally m-convex*-algebras.............. 5

Lindsay Nathan Childs and Frank Rimi DeMeyer, On automorphisms of separable algebras ...................................

Charles L. Fefferman, A Radon-Nikodym theorem for finitely additive set

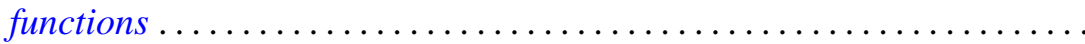

Magnus Giertz, On generalized elements with respect to linear

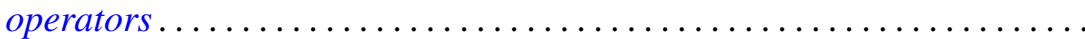

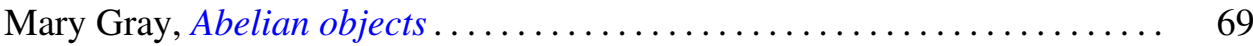

Mary Gray, Radical subcategories.............................. 79

John A. Hildebrant, On uniquely divisible semigroups on the two-cell . . . . . 91

Barry E. Johnson, AW*-algebras are $\mathrm{QW}^{*}$-algebras ............... 97

Carl W. Kohls, Decomposition spectra of rings of continuous functions . . . . 101

Calvin T. Long, Addition theorems for sets of integers .............. 107

Ralph David McWilliams, On $w^{*}$-sequential convergence and quasi-reflexivity ................................... 113

Alfred Richard Mitchell and Roger W. Mitchell, Disjoint basic

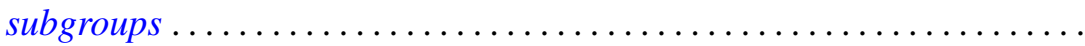

John Emanuel de Pillis, Linear transformations which preserve hermitian and positive semidefinite operators .

Qazi Ibadur Rahman and Q. G. Mohammad, Remarks on Schwarz's lemma

Neal Jules Rothman, An $L^{1}$ algebra for certain locally compact topological semigroups ...

F. Dennis Sentilles, Kernel representations of operators and their adjoints ...

D. R. Smart, Fixed points in a class of sets

K. Srinivasacharyulu, Topology of some Kähler manifolds

Francis C.Y. Tang, On uniqueness of generalized direct decompositions .

171 Albert Chapman Vosburg, On the relationship between Hausdorff dimension and metric dimension . 